\title{
La evaluación docente universitaria: retos y posibilidades*
}

${ }^{10} 49$

\section{University Teacher \\ Evaluation: Challenges \\ and Possibilities \\ A avaliação \\ docente universitária: \\ desafios e \\ possibilidades}

Ligia Ochoa Sierra** Orcid: https://orcid.org/0000.0002-8761-4819

Constanza Moya Pardo*** Orcid: https://orcid.org/0000-0003-3031-6594

\section{Para citar este artículo}

Ochoa Sierra, L. y Moya Pardo, C. (2019).

La evaluación docente universitaria: retos y posibilidades. Folios, 49, 41-60. doi: 10.17227/Folios.49-9390

* La investigación que a continuación se presenta fue seleccionada en una convocatoria de investigación de la Facultad de Ciencias Humanas de la Universidad Nacional y, en consecuencia, financiada por dicha entidad.

* Doctora en Lingüística (Universidad Autónoma de Madrid), doctora en Educación (UNED), magíster en Lingüística española (Instituto Caro y Cuervo). Profesora titular del Departamento de Lingüística de la Facultad de Ciencias Humanas de la Universidad Nacional de Colombia.

Correo electrónico: lochoas@unal.edu.co.

** Doctora en Ciencias de la Educación de la Universidad Tecnológica de Pereira. Estudios de Doctorado en Filología Española (UNED). Magíster en Lingüística Española (Instituto Caro y Cuervo) y Licenciada en Idiomas (Universidad Pedagógica Nacional). Profesora asociada Departamento de Lingüística de la Facultad de Ciencias Humanas de la Universidad Nacional de Colombia.

Correo electrónico: mcmoyap@unal.edu.co 


\title{
Resumen
}

En este artículo se presentan los resultados de una investigación en torno a las percepciones que tienen estudiantes y profesores acerca de la evaluación que semestralmente realizan los estudiantes a los docentes. Se utilizó una metodología cualitativa a través de una entrevista a grupos focales. Los resultados muestran que se siente malestar frente a la evaluación tanto de parte de profesores como de estudiantes; dicho malestar se refleja en bajos niveles de participación y receptividad de los datos que los formularios de evaluación aportan y una desmotivación generalizada hacia este proceso. Como conclusiones importantes se destaca la necesidad de contrastar las evaluaciones docentes con una autoevaluación tanto del profesor como del estudiante, conciliar las expectativas de docentes y alumnos frente a la evaluación, emprender procesos pedagógicos con profesores y estudiantes para tomar conciencia de la importancia de la evaluación.

\section{Palabras clave}

percepciones; representaciones; evaluación docente; universidad

\begin{abstract}
In this paper we present the results of a research on the perceptions of students and teachers about the teacher evaluation required of students every semester. A qualitative methodology was used by means of a focus group interview. Results show that both teachers and students feel uncomfortable with the evaluation; this discomfort is reflected on low levels of participation and receptivity of the information provided by the evaluation forms and a general lack of motivation regarding this process. As a conclusion, it is worth noting the need to contrast the teacher evaluations with a self-evaluation of both the teacher and the student, to reconcile the expectations of teachers and students about the evaluation, and to undertake pedagogical processes with the teachers and students in order to become aware of the importance of evaluation.

Keywords

perceptions; representations; teacher evaluation; university
\end{abstract}

\section{Resumo}

Neste artigo apresentamos os resultados de uma pesquisa em torno das percepções dos estudantes e professores sobre a avaliação semestral realizada sobre os docentes. Utilizamos uma metodologia qualitativa através de uma entrevista a grupos focais. Os resultados assinalam que a avaliação gera desconforto tanto nos professores quanto nos estudantes; esse desconforto está refletido nos baixos níveis de participação e receptividade dos dados que as fichas de avaliação coletam, além de uma evidente desmotivação em geral durante este processo. Como conclusões importantes destacamos a necessidade de contrastar as avaliações docentes com uma autoavaliação tanto do professor quanto do estudante, conciliar as expectativas de professores e alunos perante a avaliação, assim como empreender processos pedagógicos com professores e estudantes para uma tomada de consciência sobre a importância da avaliação.

\section{Palavras-chave}

percepções; representações; avaliação docente; universidade 


\section{Introducción}

Aunque algunas instituciones tienen mejor regulado el proceso de la evaluación docente que otras, puede afirmarse que esta es una práctica común en todas las universidades colombianas: semestralmente los alumnos evalúan a sus profesores. Si bien se estipula que la finalidad de la evaluación es valorar el trabajo hecho y mejorar las prácticas docentes, muchas instituciones usan esta información para sancionar a los profesores, para rendir cuentas y no como insumos para planes de mejoramiento. La perspectiva de considerar al profesor como responsable de los resultados de los estudiantes también es frecuente y muy cuestionable, pues como lo señala Álvarez (s.f., p. 18), citando a Cardemil (1991) "los resultados que obtienen los alumnos son efectos de múltiples factores, uno de los cuales sería el docente".

Estas miradas de la evaluación han llevado a que profesores y alumnos tengan distintas percepciones al respecto. La investigación que a continuación se presenta indaga sobre qué piensan docentes y estudiantes de la evaluación docente, qué motiva a los estudiantes a evaluar o no, qué motiva a los profesores a atender o no las evaluaciones, qué factores determinan la evaluación, qué percepción tienen acerca del formato utilizado y de la mecánica misma de la evaluación.

Las percepciones son representaciones sociales que se construyen en interacción con otros en un contexto específico. Para Moscovici (1985), son formas de pensamiento y de conocimiento individual pero matizado por la experiencia social. Se trata, por tanto, de sistemas cognitivos que se construyen para dar cuenta del entorno en que se vive y que a su vez son producto de él. De acuerdo con este investigador, lo que sabe, cree, ve y siente un actor social resulta esencial para comprender distintas esferas de la vida social. La información que proporciona una representación es un insumo decisivo para comprender las prácticas sociales.

En relación con la evaluación, las representaciones individuales de los actores involucrados reflejan la manera de percibir el fenómeno, el sistema de conocimientos, valores y creencias asociados a este.
Conocer dichas representaciones es adentrarse en el fenómeno de la evaluación desde los propios participantes, quienes tienen una mirada del problema, pues asumen una posición y actúan conforme a ella.

Acercarse a las percepciones de una comunidad determinada permite entender de qué manera sus miembros interpretan su entorno, qué significado les dan a las acciones que realizan y a las actividades en las que participan. Una vez sacadas a la luz es posible determinar sus motivaciones y qué factores determinan su accionar.

Se buscó, entonces, identificar las percepciones de profesores y estudiantes acerca de la evaluación docente que semestralmente realizan los estudiantes, ver los puntos de coincidencia y en los que discrepan, todo ello con el supuesto de que conocer estas percepciones contribuye a mejorar tanto los procesos evaluativos como, en general, los educativos.

En la revisión bibliográfica se encontró que, si bien la evaluación docente universitaria es una práctica frecuente que aparece en Latinoamérica por influencia del modelo norteamericano hacia la década de 1950, son escasas las investigaciones en torno al proceso mismo de evaluación y a los resultados obtenidos. Lo que se encuentra en la bibliografía es la descripción de los procesos adelantados por las universidades, tal es el caso del documento de la Universidad del Rosario (2012), el de la misma Universidad Nacional de Colombia (Echeverry, 2011) o el presentado por Vásquez y Gabalán (2012), a propósito de la evaluación de docentes de posgrados.

Hay también investigaciones que proponen modelos de evaluación o formatos de evaluación; por ejemplo, el trabajo de Zambrano, Meda y Lara (2005). Estos investigadores elaboraron un instrumento objetivo, lo aplicaron a 2325 estudiantes de los cursos del Departamento de Psicología Básica y correlacionaron los resultados obtenidos en la aplicación con una autoevaluación por parte de los estudiantes. En el análisis estadístico, se determinó que, en general, los factores del cuestionario de desempeño docente tuvieron una correlación positiva con respecto a la autoevaluación de los estudiantes, 
es decir, que las valoraciones que los estudiantes dieron a sus profesores en cada factor aumentan, al igual que las valoraciones que se dieron a sí mismos.

Hay también trabajos generales acerca de la evaluación en la educación superior como el de Fuentes y Herrero (1999) quienes elaboran una propuesta para la autoevaluación docente; el de Andión (2007), quien propone un modelo de corte cualitativo; el de Villa (2008), quien plantea un modelo basado en estándares de calidad, o el de Tejedor (2009), quien hace interesantes aportes teóricos y metodológicos sobre este objeto de conocimiento.

Sobre las percepciones, solo se encontró en la revisión bibliográfica una investigación realizada por Fernández, Mateo y Muñiz (1996) que indaga acerca de la valoración que hacen los docentes de la evaluación realizada por los alumnos. En ella se encuentra que los docentes valoran más positivamente la evaluación cualitativa que la cuantitativa, hacia la que tienen reparos.

La ausencia de una investigación como la que se propone fue la justificación más importante para realizar este trabajo, junto con la necesidad de mirar analíticamente las percepciones de los protagonistas educativos frente a la evaluación universitaria.

\section{Marco teórico}

La evaluación es un proceso consustancial al aprendizaje en cuanto es un insumo para verificar su desarrollo y adquisición. Se evalúa tanto el proceso en sí, como los resultados, y en este sentido la distinción entre evaluación formativa y sumativa resulta necesaria. La formativa o cualitativa tiene que ver con el seguimiento a los procesos a través de procedimientos como la entrevista, la observación, los diarios de campo; proporciona información permanente del proceso educativo. Por su parte, la evaluación sumativa o cuantitativa mide los resultados obtenidos en un programa académico o en una clase determinada, a través de test o pruebas objetivas con procedimientos matemáticos y estadísticos.

De otra parte, de acuerdo con los agentes que intervienen en la evaluación, se distingue entre la autoevaluación, la heteroevaluación y la coevaluación. Para el caso que nos ocupa, la primera la realiza el propio profesor, la segunda se lleva a cabo con pares, es decir, la que realizan colegas, y la última es la que hacen los estudiantes.

La evaluación se hace tanto para verificar los aprendizajes como para evaluar la enseñanza; en ese sentido, el protagonista de la evaluación no solo es el estudiante sino el profesor, el coordinador o director, la institución educativa y, en general, el sistema educativo. La evaluación docente es, por consiguiente, una de las aristas de la evaluación educativa impensable sin las otras aristas. Es preciso, como lo señala Villa (1985, p. 92), evaluar al profesor en el marco del sistema educativo:

La evaluación del profesor aun considerando a este como la principal variable, resulta al menos insuficiente. El profesor es una variable importante dentro de un sistema de elementos mutuamente interrelacionados. Por consiguiente, la evaluación del profesor y del (de los) rol(es) que desempeña deben evaluarse teniendo en cuenta su encuadre dentro del sistema. El estudio aislado de la variable profesor, sin referencia a un marco más amplio, desvirtúa el resultado de la investigación.

La evaluación docente solo es posible cuando:

a. Todos los miembros participan en la conformación del modelo de evaluación. Para lograr la participación activa del profesor es necesario que él comprenda el propósito y la justificación de la evaluación; solo así se logra la motivación necesaria para llevar a cabo con éxito el proceso emprendido.

b. Los miembros y sus funciones son evaluados dentro del sistema. Los profesores son evaluados no solo en términos de las competencias que adquieren sus estudiantes, sino frente al compromiso que estos tienen con su formación y el que tiene el programa con estudiantes y profesores.

c. Los criterios e indicadores de evaluación son claros y pertinentes al objeto evaluado. 
d. La evaluación se convierte en un insumo importante para cada uno de los miembros de la comunidad, pues permite identificar vacíos y problemas del sistema, realizar cambios, establecer planes de mejoramiento, combatir las desigualdades que puede generar el sistema, tomar decisiones en distintos ámbitos, asignar recursos, comprender la vida universitaria, entre otros.

Diversos investigadores (Fernández, Mateo y Muñiz, 1996; Villa, 2008; Tejedor, 2009) han resaltado la importancia de la evaluación docente hecha por los estudiantes, ya que se ve en ella una oportunidad para "detectar los puntos fuertes y débiles de la enseñanza impartida, y con ello, inferir las vías para realizar avances en el perfeccionamiento docente" (Fernández, Mateo y Muñiz, 1996, p. 171).

Es, además, una fuente de información principalmente para el mismo docente, quien puede ver cómo lo perciben sus estudiantes, cómo va el proceso que está realizando, qué debilidades hay y qué fortalezas tiene; en fin, para medir la eficacia $y$ pertinencia de su trabajo con miras a encontrar alternativas adecuadas. Pero para que sea un insumo adecuado, es preciso que el profesor, por un lado, participe en la construcción del instrumento y sepa qué y cómo se le evalúa, y por otro, tenga también la oportunidad de hacer su autoevaluación y de exigir la autoevaluación del estudiante. Muchos docentes se quejan de que son evaluados por estudiantes que apenas asisten o que han puesto muy poco de sí en los procesos de aprendizaje. Así mismo, es importante que el profesor conozca las evaluaciones a tiempo y fácilmente. Suele ocurrir que acceder a los resultados es tan engorroso que el docente termina desistiendo de verlos.

La evaluación es también un insumo para las políticas institucionales de formación docente. Vista así la evaluación, los datos cuantitativos y cualitativos de la evaluación docente, teniendo como fuentes de información los alumnos y el propio profesor, les permitirá a ambos actores educativos reflexionar sobre las prácticas docentes, comprenderlas y valorarlas. Para ello es preciso que el análisis considere el contexto en el que se desarrolla el trabajo, los recursos con los que cuenta el docente y los apoyos que ha recibido.

La evaluación depende de lo que se espera para el nivel educativo en el que se aplica; no es lo mismo evaluar al docente de primaria que al de la universidad, pues hay perfiles y funciones distintas, asignados a unos y otros. De acuerdo con la Ley 30 de 1992, algunos de los objetivos de la educación superior en Colombia son:

- Profundizar en la formación integral de los colombianos [...] capacitándolos para cumplir las funciones profesionales, investigativas y de servicio social que requiere el país.

- Trabajar por la creación, el desarrollo y la transmisión de conocimiento en todas sus formas y expresiones, y promover su utilización en todos los campos para solucionar las necesidades del país.

[...]

- ser factor de desarrollo científico, cultural, económico, político y ético a nivel nacional y regional. $[\ldots]$

- Promover la formación y consolidación de comunidades académicas y la articulación con sus homólogas a nivel internacional. (s.p.).

Desafortunadamente, la evaluación docente universitaria en distintas latitudes, no solo latinoamericanas, ha estado más relacionada con los procesos de renovación de contratos o de acreditación que con los procesos de mejoramiento, lo que ha tergiversado su naturaleza, pues se evalúa no para transformar las prácticas, si es necesario, o para consolidarlas, sino para sancionar. Stobart (2010) muestra que la evaluación en los últimos tiempos se utiliza con fines de rendición de cuentas para medir la eficacia de las instituciones. De esta manera se reduce su impacto a la obtención de resultados, y se descuida su propósito último: el aprendizaje.

Una evidencia de que la evaluación está pensada más en términos de resultados que de procesos consiste en que no es permanente ni genera una retroalimentación. Al respecto, Villa (1985, p. 354) 
afirma que "una evaluación realizada única y exclusivamente al final de un proceso o programa no es más que una respuesta burocrática a un sistema”.

De otra parte, como lo señala Arbesú (2004, p. 865) las evaluaciones docentes "poco reflejan la complejidad de las prácticas educativas o lo que aprendió el alumno y, difícilmente, dan cuenta del impacto que el profesor tuvo en las formas de pensamiento de los estudiantes".

Villa (1985, citado por Álvarez, s.f., p. 4), plantea la necesidad de que "antes de establecer un sistema de evaluación del profesor hay que clarificar la definición de la tarea docente, para poder delimitar qué hay que evaluar, así como el procedimiento más adecuado para ello". Por su parte, Arbesú (2004) propone la participación de los docentes en la construcción de un instrumento de evaluación.

Stobart (2010, p. 24) señala que en toda evaluación es necesario responder tres preguntas: “¿Cuál es la finalidad principal de esta evaluación?, ¿se ajusta la forma de evaluación a la finalidad de la misma?, ¿se consigue su objetivo?". El mismo autor indica que es necesario reflexionar acerca del uso que se hace de dichas evaluaciones:

[...] que una evaluación sea o no válida no solo depende de lo bien que mida lo que se somete a examen, sino también de las interpretaciones que se hagan de los resultados. Un test bien construido se invalida si no se entienden o malinterpretan los resultados. Muchos de los abusos de la evaluación surgen de lo que se lee en los resultados y de las consecuencias de ello. En el contexto de unas ambiciones más limitadas, tenemos que hacer unas inferencias más cautas. (p. 203).

Villa (1985, p. 74), por su parte, afirma que debe analizarse de manera muy cuidadosa el formato que se aplique al proceso evaluativo para que pueda realmente ser útil: "El feedback para que pueda ser útil, debe cumplir unos requisitos: que sea específico, concreto, inmediato, con posibilidad real de cambio, no amenazante ni formulado de modo agresivo".
Estos formatos deben ser objeto de pilotaje y de revisiones continuas.

La evaluación es un proceso que no solo "mide objetivamente lo que hay, sino que crea y configura lo que mide" (Stobart, 2010, p. 11). En ese sentido, los formatos de evaluación determinan en gran medida las evaluaciones, ya que proveen un cierto tipo de información, en ocasiones, en detrimento de otras.

En la revisión bibliográfica se encontró que los cuestionarios usaban distintos factores para evaluar la docencia. A modo de ejemplo se presentan cuatro propuestas, todas fundamentadas en la investigación.

Zambrano, Meda y Lara (2005, p. 65) elaboraron un formato con 11 factores: autoevaluación del alumno; cumplimiento del programa; dominio, calidad y pertinencia del profesor; utilización de materiales didácticos; evaluación de las instalaciones; apoyo bibliográfico; asistencia y permanencia del profesor; habilidades didácticas; calidad de los materiales; responsabilidad del alumno, y habilidades de comunicación.

Por su parte, Casero (2008, p. 38) propone las siguientes dimensiones: cumplimiento de las obligaciones, infraestructura, programa, conocimiento/ interrelación de la materia, metodología, materiales, actitud del profesor (respetuoso, accesible), evaluación, prácticas (correlaciona teoría y práctica), satisfacción (del estudiante).

Tejedor (2009, p. 96) agrupa los ítems en cuatro dimensiones: a) planificación de la docencia, b) desarrollo de la docencia, c) resultados (consecución de objetivos y satisfacción de los estudiantes), y d) mejora e innovación (formación recibida, innovación en proyectos, innovación en materiales).

El instrumento en el que se basa la presente investigación consta de 19 preguntas que tienen opción de respuesta cerrada y 2 con pregunta abierta. Una de las preguntas cerradas indaga por el desempeño global del docente. Estas preguntas se agrupan en tres dimensiones: 
a. Humana, social y ciudadana. A través de esta dimensión se busca reconocer si el docente conoce, aplica y enseña los principios y valores sociales e institucionales, así como los derechos y deberes fundamentales que tiene como persona y como ciudadano, en un marco de respeto hacia el prójimo y las instituciones, conocedor de lo que la sociedad espera de él.

b. Pedagógica. Es la capacidad de hacer uso de herramientas, estrategias y saberes disciplinares aplicados al proceso de enseñanza/ aprendizaje, que orientan al estudiante en la construcción de sus propios conocimientos, actitudes y valores; de este modo, el estudiante desarrolla habilidades profesionales y éticas, y se genera una formación integral y de larga vida.

c. Disciplinar-profesional. Es la demostración de interés por la actividad académica e investigativa y por actualizar los conocimientos disciplinares o profesionales de manera permanente, con el fin de dominar los temas relacionados con su disciplina y saber, compartiendo sus conocimientos, vivencias, experiencias, y reflexiones con sus alumnos, convirtiéndose de este modo en un modelo de actuación profesional (Echeverry, 2011).

Los formatos anteriores tienen como común denominador el hecho de que la evaluación se concibe desde un modelo de evaluación centrado en los rasgos del profesor, específicamente en tres características: cualidades académicas (dominio conceptual, estrategias didácticas, uso de recursos), cualidades personales (puntualidad, responsabilidad) y cualidades sociales (interacción con los estudiantes, respeto hacia ellos). Así mismo las propuestas de cuestionarios apuntan en mayor o menor medida a los momentos de la docencia: planificación, desarrollo y evaluación. En todos los formatos, se espera que el docente motive a los alumnos y los apoye en la construcción de conocimiento y en el desarrollo del pensamiento crítico.
Solo en el formato de Zambrano, Leda y Lara (2005) se correlaciona el desempeño docente con el del alumno, aspecto que consideramos fundamental en la evaluación, puesto que del compromiso y actitud del alumno depende en gran parte el éxito de la actividad docente. Tampoco aparece la opción de que el profesor presente su voz, reflexione sobre su quehacer.

\section{Metodología}

Para dar cuenta de las percepciones de profesores y estudiantes acerca del objeto de estudio seleccionado, se utilizó una metodología cualitativa. La investigación como totalidad usó dos procedimientos metodológicos: una encuesta virtual de preguntas cerradas y una entrevista que se aplicó en un grupo focal.

Con el primer instrumento se hizo una exploración para identificar la percepción de la población con relación a las vivencias experimentadas sobre la evaluación. El cuestionario de la encuesta virtual de los estudiantes fue el siguiente: ¿Conoce el formato de evaluación docente? ¿Evalúa semestralmente a sus docentes? ¿Le parece importante evaluar a los docentes? ¿El formato permite evaluar qué enseña el docente y con qué calidad? ¿El formato permite evaluar qué impacto tuvo en el estudiante lo que enseña el docente? ¿El formato permite evaluar qué grado de compromiso tuvo el estudiante? ¿La evaluación docente tiene algún impacto en los procesos de mejoramiento académico? ¿Hace comentarios u observaciones a sus docentes? ¿Cree que situaciones de anormalidad académicas inciden en la evaluación de los docentes? ¿Sus resultados académicos determinan la evaluación que otorga a sus docentes?

La encuesta virtual dirigida a los profesores se construyó con base en los siguientes interrogantes: ¿Conoce el formato de evaluación docente? ¿Ha estado satisfecho(a) con sus evaluaciones docentes? ¿Tiene en cuenta los resultados de la evaluación cuantitativa que le hacen los (las) estudiantes? $¿$ Tiene en cuenta las observaciones cualitativas que le hacen los (las) estudiantes? ¿Tiene acceso a las evaluaciones semestrales? ¿Los resultados académicos 
de los (las) estudiantes determinan la evaluación que ellos (ellas) le otorgan? ¿El formato permite evaluar qué enseña el (la) docente y con qué calidad? ¿El formato permite evaluar qué impacto tuvo en el (la) estudiante lo que enseña el (la) docente? ¿ El formato permite evaluar qué grado de compromiso tuvo el (la) estudiante? ¿Está de acuerdo con el proceso de evaluación docente que hacen los (las) estudiantes?

Una vez hecha la tabulación de los datos, se consideró oportuno indagar acerca de las razones de las respuestas cerradas y de otros temas que no se pudieron preguntar con un cuestionario de preguntas cerradas, así como acceder a los juicios valorativos sobre el modelo evaluativo. Se decidió, en consecuencia, realizar una entrevista en profundidad con grupos focales. Los resultados que se presentan en este artículo son los correspondientes a los datos de estos grupos.

Se diseñó una encuesta semiestructurada con una secuencia de temas y algunas preguntas abiertas sugeridas, que se aplicó a 35 estudiantes y a 8 profesores. Los temas y las preguntas se centraban en aspectos como: concepto de evaluación, normatividad sobre la evaluación, formato utilizado, posición frente a la evaluación, momento oportuno para evaluar, tipo de evaluación preferida (cuantitativa o cualitativa), expectativas frente a la evaluación docente, factores externos asociados con ella, grado de satisfacción frente a este proceso, sentimientos que despierta y recomendaciones. A los estudiantes se les preguntó también acerca de lo que es un buen o mal profesor, y a los docentes, si se sentían conformes con el mecanismo de la evaluación. Las entrevistas se grabaron y luego fueron transcritos apartados que se consideraron relevantes para el estudio.

La población estuvo constituida por estudiantes y profesores de una Facultad de Ciencias humanas de una universidad pública. Se tuvieron como criterios de selección el que estuvieran vinculados activamente a una carrera de la facultad y que desearan participar en la investigación. Ambas poblaciones fueron informadas acerca del propósito de la investigación. La participación fue voluntaria.
Los alumnos tenían como media de edad 21 años. Se seleccionaron tres estudiantes por cada uno de los siguientes departamentos de la facultad de Ciencias Humanas: antropología, filosofía, geografía, historia, lenguas extranjeras, lingüística, literatura, psicología, trabajo social, sociología.

En relación con el género, hubo mayor representatividad de las mujeres (61\%) frente a los hombres. Los estudiantes estaban en distintos semestres: desde segundo, hasta estudiantes de undécima y décima segunda matrícula.

La muestra de profesores estuvo conformada por docentes de los departamentos de lingüística, lenguas extranjeras, antropología, psicología, geografía, filosofía, trabajo social e historia. La mayoría de los docentes eran de tiempo completo y tenían como categoría la de profesor asociado. Hubo una representación igual de hombres y mujeres.

Debido a problemas de disponibilidad, los profesores se conformaron en dos grupos de 4 docentes cada uno; los alumnos, en grupos de 7 estudiantes.

Los datos fueron analizados desde la metodología denominada análisis de contenido, a partir de los tópicos ya señalados. Inicialmente, ambas investigadoras hicieron un análisis exploratorio del material grabado y transcrito con el fin de establecer ciertos patrones regulares en los datos; luego, cada una estableció unas categorías de análisis que luego consensuaron y verificaron regresando de nuevo a la grabación y transcripción. Todo el procedimiento de análisis se hizo manual.

\section{Resultados}

El análisis de los datos se ilustra en la red semántica de la figura 1, la cual se centra en dos categorías: el modelo de evaluación y la evaluación misma. 

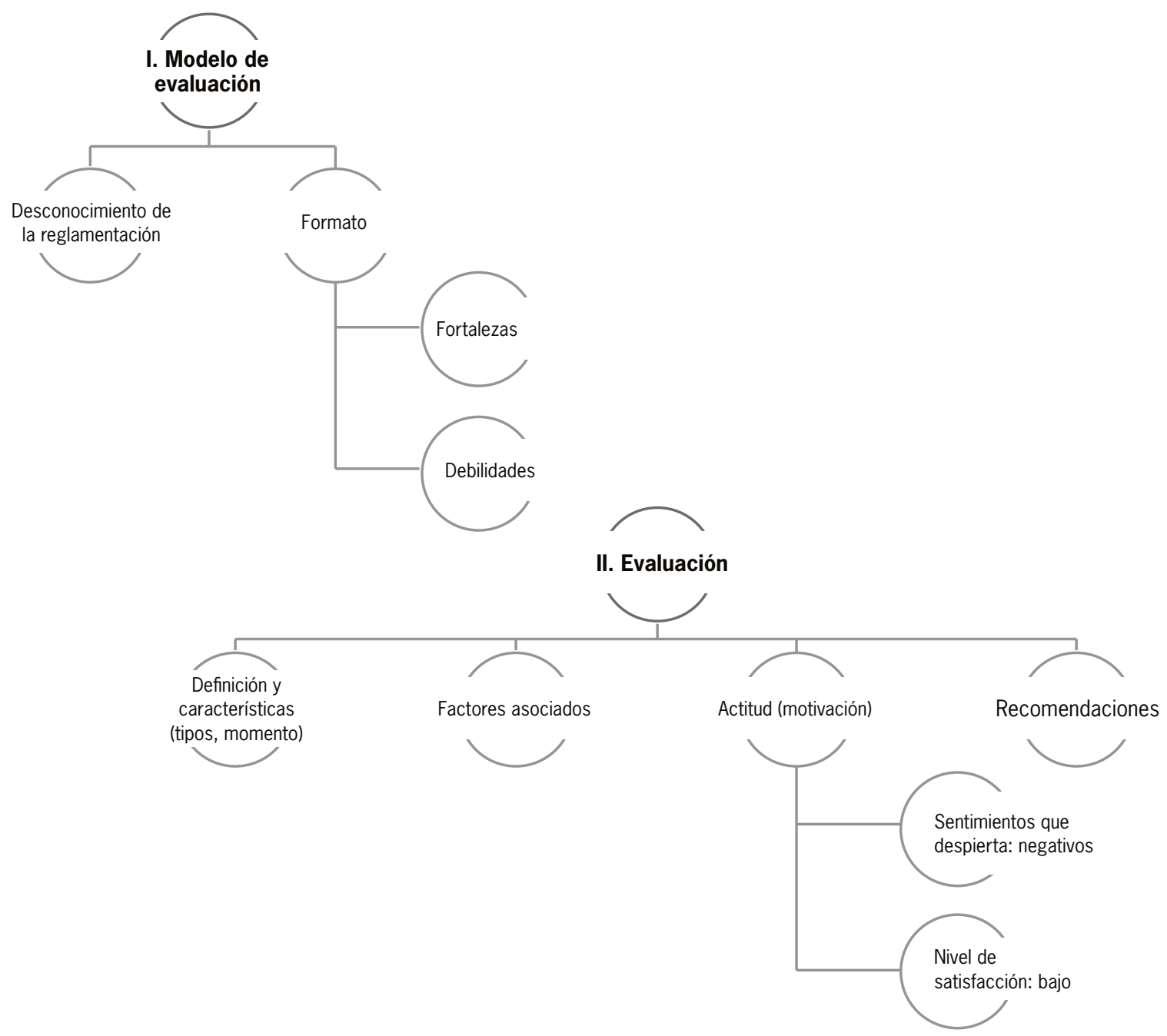

Figura 1. Aspectos considerados para el análisis

Fuente: elaboración propia.

A continuación, se desglosa esta red en cada una de las poblaciones. Se presentan los resultados de los alumnos, luego el de los profesores y, posteriormente, se hace una comparación entre ambos.

\section{Alumnos}

La discusión en el grupo focal mostró que hay un total desconocimiento por parte de los estudiantes sobre la reglamentación de la universidad, relacionada con la evaluación docente. Los estudiantes señalaron que no la conocían. Frente al formato con el que se evaluaba al docente, señalaron que sí lo conocían. No obstante, ningún estudiante dio cuenta de su estructura en forma completa y adecuada, sino que se señalaron aspectos que recordaban. El de mayor recordación fue el de la metodología del docente (factor pedagógico) y seguido de la puntualidad en la entrega del programa, de las evaluaciones y de las mismas clases (factor personal).

Sobre las fortalezas del formato, la mayoría de alumnos destacó la facilidad y comodidad de llenarlo, al ser virtual, y su sencillez. Se resalta también el que fuera anónimo, pues permitió libertad de opinión. Un número pequeño de estudiantes destacó que era muy completo, es decir, evaluaba lo que tenía que evaluar. Un número importante de alumnos señaló que no sabía, no recordaba o no lo conocía bien. 
Frente a los cambios que harían al formato, la mayoría de entrevistados respondió que no harían ninguno. Los que lo cuestionaban indicaron que la principal crítica residía en que el formato no discriminaba la especificidad de las asignaturas; se señaló que había unas particularidades en cada área y que no era posible recuperarlas en la evaluación actual. Se cuestionó también que no hubiera preguntas relacionadas con el compromiso del estudiante y la autoevaluación de estudiantes y profesor.

Son interesantes estos últimos comentarios que apuntan a la necesidad de escuchar también la voz del profesor, a pedir una mayor responsabilidad por parte de los estudiantes al momento de evaluar y tener en cuenta las diferencias de asignaturas. Obsérvese que los cambios no apuntan al contenido mismo del formato.

En relación con la definición de evaluación, las respuestas se centraron en tres aspectos. En primer lugar, en el acto mismo de lo que se hace al evaluar: analizar, calificar, opinar o presentar una percepción, dar una valoración, informar, hacer un seguimiento. En segundo lugar, en lo que se hace cuando se evalúa, es decir, juzgar, medir, determinar la calidad, hacer un balance, criticar, revisar un proceso. En tercer lugar, en el objeto de la evaluación: habilidades y competencias, desempeño, fortalezas y debilidades, aspectos positivos y negativos.

Algunos entrevistados resaltaron el hecho de que la evaluación se realiza en un contexto determinado (un tiempo y un espacio; por ejemplo, un semestre en una universidad pública, en un programa o en una asignatura específica). Se destaca también la necesidad de tener en cuenta unos criterios o instrumentos, unos puntos de partida y de llegada o la relación entre lo que se esperaba y lo que resultó.

Las respuestas fueron tanto generales (qué es evaluar en términos generales) como particulares al acto educativo y más específicamente al acto mismo de la docencia universitaria.

La mayoría de estudiantes dijo estar de acuerdo con evaluar a sus docentes, pues sienten que tienen la competencia para evaluarlos, ya que son los receptores del proceso educativo, lo que les da el derecho a hacerlo. Esta evaluación permite tener la perspectiva del estudiantado. Estudiantes que no evalúan señalan que no lo hacen porque sienten que la evaluación es inútil, porque les da pereza, se les olvida, falta de tiempo o problemas con la plataforma. Aparece también como una razón para no evaluar el que se considere que este proceso debe hacerse en forma presencial y oral como un medio de comunicación con el docente.

Sobre el tipo de evaluación que prefieren, la mayoría de alumnos afirmó que la evaluación cualitativa, ya que permite hacer comentarios más precisos, desarrollar, profundizar o justificar una respuesta cerrada. No obstante, se resalta de la evaluación cuantitativa su objetividad.

Frente al momento oportuno del semestre para evaluar, la mayoría señala que al final del semestre pues ya se conoce al profesor y se ha vivido el proceso educativo; sin embargo, algunos estudiantes señalan que a la mitad del semestre para que el profesor pueda hacer los cambios pertinentes a tiempo.

En cuanto a la pregunta de qué espera de la evaluación docente, la mayoría de entrevistados señala que espera que sea útil, que sea tenida en cuenta, que genere cambios en los docentes y que mejore la calidad educativa. Se espera también que sea objetiva.

A propósito de los factores externos que podrían incidir a la hora de evaluar a los docentes, las respuestas en esta pregunta apuntaron a tres cuestiones en el siguiente orden: anormalidad académica (bloqueos y paros), notas o resultados de los estudiantes, y relaciones interpersonales con docentes, especialmente las malas relaciones que afectan la objetividad del estudiante, lo que le genera poca empatía, rencores, emociones negativas, resentimientos y malas evaluaciones para los docentes.

En relación con los paros, los entrevistados consideraron que los profesores se ausentan y distancian de estos movimientos sociales, lo que refleja, según ellos, falta de compromiso con la universidad y la educación pública. Se cuestionó la falta de comunicación continua con los estudiantes. También, se señaló como un factor que determina la evaluación docente el hecho de que los tiempos se 
acortan y los docentes intentan hacer en tres meses lo que se tiene pensado para cuatro, lo que genera mal manejo didáctico y sobrecarga en el estudiante; esto, a su vez, no permite los logros esperados. Tampoco lo facilita el hecho de dejar temas a un lado o tratarlos superficialmente: se truncan los procesos, generando insatisfacción en el estudiante. La evaluación al docente, entonces, está mediada por la manera como enfrentó la anormalidad académica. Algunos estudiantes señalan que, si bien el profesor no es el responsable directo, el profesor pueda dar una imagen equivocada y ser evaluado conforme a ella por la situación generada.

Quedó vedada la queja de que el profesor sobrecarga al estudiante, se preocupa más por los contenidos y el programa que por el aprendizaje. Hubo crítica al profesor que se margina del proceso y a la vez al que no hace clase. Se criticó fuertemente a docentes que por todos los medios desean cumplir con el calendario previsto debido a sus compromisos, expresados en viajes y vacaciones.

Frente a las notas, un $40 \%$ de estudiantes consideró que las bajas notas son responsabilidad del profesor: porque no los motivó y porque no enseñó bien. Sienten, por tanto, que la evaluación del docente sí tiene relación con los resultados académicos de los estudiantes. Cuando se percibe que la nota fue injusta o sin justificación, la evaluación docente es negativa.

El $60 \%$ de los entrevistados adujeron que los resultados no dependen del profesor sino del esfuerzo del estudiante y de otros factores; que no hay relación entre notas obtenidas y evaluación docente porque se intenta ser objetivo, sería deshonesto y porque se ha evaluado bien a profesores con los que se ha obtenido malas notas, y al revés.

Aparecen también como aspectos importantes que inciden en la evaluación docente los siguientes: "los rumores, predisposiciones respecto a la clase o al profesor (lo que muestra alto grado de subjetividad), exceso de trabajo del profesor y de los estudiantes y el tiempo (no se dispone de tiempo para hacer la evaluación y se hace de afán)".
Para la mayoría de la población entrevistada, el grado de satisfacción frente a este proceso es bajo, debido a que se percibe que no tiene ningún impacto en el mejoramiento académico. Se aducen como razones el hecho de que los profesores continúan igual y son pocos los alumnos que evalúan, lo que demuestra que la evaluación no es tomada en serio ni por profesores ni por estudiantes; se argumentó también que la evaluación es subjetiva y poco constructiva, y que no hay un verdadero seguimiento a estas evaluaciones.

Sobre el sentimiento que despierta el proceso de evaluación docente, la mayoría señaló sentimientos negativos debido a que se percibe que no se hace nada con estas encuestas, a que el trabajo es inútil. Se usan lexemas como: "indiferencia, desesperanza, impotencia, aburrimiento, perdedera de tiempo, ganas de rajar, desahogo, ansiedad, pereza, desconcierto, incertidumbre, ira, fastidio, rabia, desmotivación, preocupación, desinterés, incomodidad, molestia”.

Unos pocos estudiantes mencionaron sentimientos positivos como "pensamiento crítico, ayuda, autonomía, democracia, tranquilidad, esperanza, interés, pertenencia, alegría, reflexión, ilusión, justo, incluyente, satisfacción, seguridad", los cuales apuntan al derecho y al deber de la evaluación.

Sobre las recomendaciones al proceso de evaluación docente realizado por los estudiantes, un alto porcentaje de los entrevistados señaló que no sabía o dijo que no tenía "ninguna" recomendación. Para los restantes, la recomendación más insistente es que tenga algún efecto o impacto en los profesores y en las decisiones de directivos, como cambiar al docente de curso, expulsarlo y estimular al buen profesor. Se pidió también "una mayor incentivación a los estudiantes para que lo hagan". Se hicieron también sugerencias sobre el formato y el proceso mismo: que sea objetivo y justo (mecanismos que reduzcan el sesgo que pueden tener los estudiantes) que no sea tan largo, "que los profesores contesten los comentarios que se les hace, sobre todo si tuvieron críticas" y "que sea obligatorio evaluar".

A propósito de lo que es un buen profesor, la mayoría de respuestas indicaron como primer aspecto que es quien maneja una metodología 
adecuada que permite, por una parte, explicar en forma clara y por otra, lograr que los alumnos lo entiendan. En segundo lugar, se destaca la pasión que muestra en su clase y la motivación que genera en los alumnos. Tercero, un buen profesor es quien entiende al estudiante, comprende sus necesidades, adecua sus procesos a las particularidades de su grupo, dialoga con ellos y facilita la discusión en clase. En cuarto lugar, se destaca que el docente domine los temas o contenidos del curso y cuestione o sea crítico con la bibliografía. Por último, se resaltan rasgos personales como paciente, calmado, con vocación, puntual, exigente, respetuoso, tolerante, comprensivo, imparcial.

Opiniones individuales señalaron que un buen profesor es quien "incentiva al estudiante a la investigación y al trabajo autónomo" y "aquel que logra chocar a sus estudiantes, crear una ruptura mental o vivencial".

Un mal profesor es todo lo contrario a lo anterior: no es objetivo, es irresponsable, "pedante, no se retroalimenta ni escucha a sus estudiantes, no se actualiza", "enseña poco o evalúa mal", "no se hace entender", "es irritable", "improvisa y llena clases sin contenidos", "no dicta lo que debe o no exige como debe", "no explica bien y no es cumplido con sus horarios o calendarios", "no se interesa si el estudiante aprende o no", "no es comprometido con la clase" "no se interesa por solventar las dudas de sus estudiantes y por aportarles claridad", es "acrítico con los contenidos", "lo esencial son las notas, no los estudiantes,", "trabaja solo por dinero, no le interesa el estudiante"; "no escucha aportes", "exige cosas que en realidad ni siquiera maneja o evalúa adecuadamente", y "se impone a la fuerza, no por la razón”.

\section{Profesores}

La mayoría de profesores conocen el formato, pero desconocen la reglamentación general de la universidad sobre la evaluación docente. Se señala que el formato tiene los siguientes aspectos: contenidos, preparación de las clases, puntualidad, entrega del programa y forma de evaluar, lo que efectivamente aparece en él.
En relación con las fortalezas del formato, las respuestas apuntaron a distintos aspectos, como al hecho de que el formato trata de evaluar lo fundamental, a que tiene preguntas abiertas y cerradas que permiten análisis estadísticos, a la claridad de las preguntas y a los mismos aspectos que evalúa: "como los relativos al programa, a la motivación, al aprendizaje"

Frente a los elementos problemáticos del formato o de su aplicación, las respuestas fueron diversas. Para unos profesores el problema de la encuesta radica en la subjetividad del estudiante, en el hecho de que son castigados los profesores más exigentes y con quien el estudiante obtiene malas notas. Para otros docentes, las preguntas abiertas se prestaron para divagación, insultos y comentarios degradantes que no contribuyen en nada al mejoramiento de la educación. Otras críticas apuntaron al hecho de que todo formato es limitado y a que no siempre evalúa lo que debe evaluar. Se cuestionó también el hecho de que el profesor no llena el formato ni puede responder o defenderse de las evaluaciones de los alumnos: se afirma que no hay un proceso de autoevaluación. Se preguntó acerca de los índices o indicadores de un criterio: “¿Cómo se sabe si el docente preparó adecuadamente la clase? ¿Si promovió la reflexión crítica?”. Se cuestiona también el efecto que puede tener una evaluación cuando no tiene representatividad. Un número reducido de profesores señaló que el formato no evalúa lo que es más importante: no lo que sabe el profesor sino "cómo organiza sus clases. Por eso, profesores con un conocimiento profundo, pero que no hacen payasadas, suelen ser mal evaluados".

Para la población de docentes, evaluar es un proceso que retroalimenta las prácticas docentes, una posibilidad de mejoramiento. Esta definición se acota ya sea al señalar su finalidad o los parámetros que se deben tener en cuenta para realizarla. Aparece también la idea de medir, ponderar, valorar y contrastar.

La mayoría está de acuerdo con la evaluación hecha por los estudiantes y tiene en cuenta las observaciones y las notas asignadas por ellos, porque a través de este medio se conocen las percepciones de los estudiantes sobre el proceso de enseñanza, y estas son esenciales para los procesos de mejoramiento 
académico, son una retroalimentación importante para el docente, quien puede cambiar, fortalecer o mantener ciertos procesos. Se destacó la idea de que la función de la evaluación es el mejoramiento y de que, si los alumnos sienten que hay problemas, hay que solucionarlos.

El $10 \%$ de los profesores que están de acuerdo con ser evaluados hicieron la salvedad de que esa evaluación no debe ser la única fuente de información sobre el desempeño docente, sino que debe complementarse con la autoevaluación y con la evaluación de pares académicos; debe ser integral $\mathrm{y}$ tener un carácter formativo no punitivo.

Los profesores que están en desacuerdo con la evaluación estudiantil señalaron que la evaluación que hace el estudiante depende mucho de la nota que obtuvo, que no la hace pensando en mejorar los procesos académicos sino en sancionar y ridiculizar al profesor, y que los resultados están condicionados por las características personales de los alumnos.

Un $5 \%$ considera que la evaluación debe ser en el aula para poder tener una retroalimentación oportuna, poder responder a las críticas y para que el evaluado asuma la posición que defiende. Para este $5 \%$ es un grave error que la encuesta sea anónima.

Para la mayoría de los docentes, ambos tipos de evaluación -cuantitativa y cualitativa-son útiles, ya que se complementan y son necesarias.

Los profesores consideran que es mejor hacer la evaluación al final del semestre pues así los estudiantes se sienten menos presionados y tienen mayor libertad.

Sobre lo que esperan de la evaluación, la mayoría de docentes pide que sea justa, que haya una correlación entre desempeños del estudiante y sus apreciaciones, y que se tome conciencia de que es solo un insumo, no puede considerarse como "la última palabra" sobre un docente. Se señala que la evaluación debería servir para mejorar los procesos didácticos. Un $40 \%$ espera que los estudiantes la realicen con responsabilidad.

Una docente pide que tenga autoría y que los estudiantes que pierden por falla no evalúen: "que no la realicen los que no asisten o van mal. También sugiero que no sea anónima, deberían aparecer los nombres".

A propósito de los factores externos que inciden en la evaluación docente, algunos profesores consideran que situaciones de anormalidad académica, calificaciones de los estudiantes, poca empatía y rumores (entre alumnos e incluso docentes que incitan a evaluar mal a un profesor) determinan en muchas ocasiones las evaluaciones docentes. El desprestigio de un profesor fomentado por un colega es una situación inadmisible en un entorno académico, no solo por razones éticas sino porque no se logra nada con esta actitud.

Los docentes dicen que no se encuentran del todo satisfechos con sus evaluaciones docentes debido a la poca participación estudiantil en las evaluaciones (el porcentaje es mínimo), lo cual no permite ver los resultados como fiables. En segundo lugar, se señala la falta de objetividad y de actitud constructiva de los estudiantes para evaluar. Frases como "mala fe", "prejuicios personales" muestran este descontento. En tercer lugar, se cuestiona el poco efecto que tiene la evaluación (positiva o negativa).

Sobre los sentimientos que despierta el proceso de evaluación docente, el que aparece con mayor frecuencia es el de desconfianza, por los pocos efectos que produce y por su poca representatividad. En menor medida se expresan sentimientos de tensión (por las consecuencias), curiosidad (por saber las opiniones de los alumnos), ambigüedad (pues se reconoce necesario, pero genera miedos), rechazo (emiten juicios que no son pertinentes al desempeño de cada estudiante), injusticia (no todo lo que dicen es verdad, no sustentan adecuadamente sus aseveraciones), incomprensión, desacuerdo (cuestionamiento respecto a lo que ellos conciben como evaluación). Se siente también impotencia al "no tener derecho a la defensa".

De lo anterior se puede concluir que para los docentes la evaluación docente es tensionante y poco objetiva.

Se profundizó en qué sentimientos genera el ser bien o mal evaluado. $\mathrm{Al}$ respecto, se señala que, 
cuando se es evaluado negativamente, se siente frustración, desilusión, injusticia, pues muchos comentarios no corresponden con la realidad. Algunos docentes señalan que si el comentario es justo, analizan las respuestas de los alumnos, las razones por las que fue evaluado de esa manera y tratan de mejorar. Las evaluaciones positivas son recibidas con alegría, entusiasmo y con cautela.

En relación con las recomendaciones al proceso de evaluación docente realizado por los estudiantes, las respuestas de los docentes apuntan a lograr mayor objetividad, rigurosidad (la responsabilidad y seriedad con la que debe realizarse) y participación del estudiantado. Se pide que cuando no haya representatividad, la encuesta no sea tenida en cuenta. Un $10 \%$ de los profesores sugiere que el alumno firme la evaluación que realiza o que se haga en el aula ("Sí, pero en presencia, como lo hacemos nosotros con ellos, no una evaluación en el anonimato; si dialogo con alguien que es anónimo, ¿cuál es el sentido de esta evaluación?").

Se señala, igualmente, que la evaluación debe ser más integral, de tal manera que los estudiantes no sean los únicos que evalúen al docente. Esta información debería cruzarse con otra que incluya el contexto más general en el que se lleva a cabo el proceso. Frente a esta última consideración, algunos docentes creen que las condiciones en las que se ejercita la docencia, condiciones que en ocasiones son adversas, afectan los procesos educativos:

Pienso, por ejemplo, en la evaluación que recibe un docente ocasional que ingresa en la tercera semana de clase, o un docente que tiene un salón de clase para 20 y recibe 30 estudiantes, o un docente que tiene clases un día en el que siempre hay problemas, o que debe dictar clases los días lunes, etc.

En relación con la pregunta de si se siente conforme con el mecanismo de la evaluación hecha a los docentes, la respuesta mayoritaria fue "no", lo que resulta sorpresivo, dado que se destaca la importancia de esta evaluación. Se señalaron como razones: la falta de divulgación y análisis de los resultados, las implicaciones laborales negativas que puede tener la evaluación, la falta de participación de los docentes en la construcción de la encuesta y la falta de impacto: no hay ni estímulos ni castigos, resultado de la evaluación. Esta última respuesta es contraria a la que dan los docentes que esperan que la evaluación no tenga ninguna repercusión laboral.

No obstante, la mayoría de docentes señalaron que tienen en cuenta las observaciones hechas por los estudiantes: acciones como enviar el programa por correo, entregar las notas oportunamente, hablar en lenguaje menos formal, disminuir el número de trabajos extraclase, mejorar las relaciones interpersonales, cumplir con las fechas pactadas y con lo establecido en el programa, se han incorporado en el desempeño docente.

\section{Comparación resultados alumnos y profesores}

Los datos muestran que hay una percepción similar frente a varios de los tópicos analizados. En lo que se discrepa es en el análisis de los temas.

Para los docentes, evaluar es esencialmente un proceso mientras que para los estudiantes es una acción para ponderar un trabajo. En ambas poblaciones, la finalidad fundamental de la evaluación docente es el mejoramiento de las prácticas pedagógicas.

Ambas poblaciones desconocen la reglamentación de la universidad sobre la evaluación docente; sin embargo, dan cuenta de manera parcial del formato utilizado.

El aspecto que más se recuerda del formato tiene que ver con la metodología del docente. Según el imaginario de los entrevistados el formato mide esencialmente aspectos didácticos. Se señala, por parte de los profesores, que el tema de los contenidos y de lo que sabe el docente está casi ausente.

Algunos profesores y estudiantes también coinciden en señalar que el formato no evalúa el grado de compromiso que tuvo el estudiante en la asignatura. Los profesores piden que se cruce el desempeño del alumno con la evaluación que otorga y que el profesor también se autoevalúe y tenga el derecho a la réplica.

Los docentes esperan de la evaluación hecha por los estudiantes, en primer lugar, que sirva para mejorar los procesos didácticos y, en segundo lugar, 
que sea justa. Mientras la mayoría de docentes pide que la evaluación estudiantil sea solo un insumo, los estudiantes reclaman mayor impacto de las evaluaciones que ellos hacen, impacto expresado en cambios y sanciones a los docentes.

Las dos poblaciones encuestadas consideran que la anormalidad académica incide en los resultados de la evaluación docente. Discrepan, no obstante, en las razones que dan: para los profesores los alumnos castigan al profesor por el paro, le atribuyen una responsabilidad que no tiene. Los estudiantes muestran una ambivalencia en cuanto a la anormalidad académica y a la responsabilidad que tienen los docentes frente a esta situación. Unos estudiantes desean que los profesores apoyen movimientos reivindicatorios, otros exigen que los profesores hagan clases en estos periodos.

Profesores y estudiantes coinciden en su sentimiento negativo frente a la evaluación: los alumnos porque sienten que no tiene ninguna repercusión y los profesores porque sienten que no es objetiva, no hay una correspondencia entre el trabajo y el resultado obtenido, y no es representativa. Ambas poblaciones señalan que la evaluación no tiene un impacto real.

Los estudiantes prefieren la evaluación cualitativa, y los profesores, ambos tipos de evaluación. Se cuestiona, por parte de los alumnos, el que los resultados que se hacen visibles suelen ser los cuantitativos (por medio de datos estadísticos del desempeño del profesor y por medio de cuadros comparativos) y no hay ningún trabajo con la información cualitativa.

Estudiantes y profesores coinciden en que el momento más adecuado para evaluar es al final del semestre, cuando ya se conoce al profesor y se ha concluido el proceso. No obstante, un menor número de entrevistados cree que debe hacerse a la mitad del semestre para que el profesor reciba una retroalimentación oportuna. Seguramente se quiere señalar la importancia de hacer un alto en el camino.

Aunque la mayoría de profesores y estudiantes concuerdan en que los resultados de los estudiantes no determinan la evaluación docente, un número significativo de profesores y estudiantes cree que sí. Ellos perciben que las bajas notas de los estudiantes son directamente proporcionales a la evaluación docente. Las razones que se aducen son distintas para cada población. Para los docentes, es un asunto de revanchismo, de falta de conciencia acerca de lo que implica la evaluación docente; para los alumnos, el hecho de ser humanos explica que cuando se siente que una nota fue injusta se castigue al profesor. De otra parte, para un número importante de alumnos, las bajas notas son responsabilidad del profesor y por ello la evaluación debe correlacionarse con los resultados de los alumnos.

A pesar de las fuertes críticas que se hace a la evaluación docente, la mayoría de la población está de acuerdo con que se haga. Profesores y estudiantes indican que es importante que los estudiantes evalúen a sus profesores, por cuanto son los directos destinatarios de sus acciones y de sus procesos formativos, $\mathrm{y}$ que ellos tienen la competencia para evaluar.

Tanto alumnos como docentes piden mayor participación estudiantil con el fin de darle mayor peso y representatividad al proceso.

\section{Discusión y conclusiones}

El análisis de los datos muestra que hay una gran desmotivación hacia la evaluación por parte de profesores y estudiantes ("el proceso no sirve para nada, no genera cambios"; "siento que es inútil ese trabajo, que los profesores no se molestan en leer los comentarios"). Es claro que para los profesores la evaluación docente tiene que ver más con factores externos que con su propia elección ("la evaluación solo se hace para controlar a los docentes y para tener cifras que mostrar") y no hay hacia ella una motivación intrínseca: los sentimientos de indefensión, amenaza, injusticia y presión que se asocian con la evaluación repercuten en la motivación ("a menudo he sido evaluado negativamente por grupos de estudiantes con prejuicios o con actitudes sesgadas por otros docentes"). Es preciso lograr una mayor identificación con la evaluación a través de la participación en la construcción del instrumento, la autoevaluación y el derecho a la réplica. Este proceso debe ser instaurado 
en las instituciones educativas universitarias y ser socializado a los miembros de la comunidad.

Tanto en la bibliografía (Fuentes y Herrero, 1999; Tejedor, 2009) como en las respuestas de algunos entrevistados se señala la necesidad de que las evaluaciones docentes se contrasten con una autoevaluación por parte del estudiante y con los desempeños obtenidos por él ("sugiero cruzar la evaluación con el promedio de cada estudiante"). Se cuestiona que estudiantes que pierden por fallas un curso o pierden la asignatura con bajas notas evalúen a sus docentes, pues no tienen la autoridad ni moral ni académica para hacerlo ("me parece terrible que evalúen estudiantes que no asisten a clase y que no trabajan ni se preocupan por su aprendizaje. ¿Son idóneos para evaluar?).

A diferencia de lo señalado por Fernández, Mateo y Muñiz (1996, p. 170), quienes afirman que "los profesores universitarios tienden a valorar más favorablemente los aspectos formativos de la evaluación de su calidad docente que aquellos otros de índole sumativa", los docentes entrevistados destacan el valor de ambos tipos de evaluación, para dar una imagen más completa del proceso seguido.

Se resalta el valor de la evaluación formativa, por cuanto permite el mejoramiento de las prácticas docentes, el cual se logra con la autorreflexión, la lectura y el diálogo entre los distintos actores involucrados (Arbesú, 2004). Solo la autorreflexión permite al docente tener una autoconciencia acerca de su trabajo y de sí mismo como docente.

La necesidad de complementar la evaluación que realizan los estudiantes con una autoevaluación por parte del profesor, aspecto señalado por algunos profesores y alumnos entrevistados ("es necesaria una evaluación que tenga en cuenta la mirada del propio profesor"), ha sido resaltada por diversos investigadores entre los que se destacan Fuentes y Herrero (1999), Arbesú (2004), Tejedor (2009), Villa (2008), entre otros.

Los datos muestran, además, que profesores y estudiantes tienen expectativas distintas frente a la evaluación docente ("la evaluación no produce ningún efecto" vs. "no debe tener implicaciones laborales negativas"). Es necesario conciliar estas expectativas. Ambos protagonistas del acto educativo han de conocer qué se espera de la evaluación docente, cuáles son sus objetivos, sus límites y posibilidades. Si se convoca a una persona para que evalúe y esta tiene entre sus expectativas que su comentario será tenido en cuenta para mantener o expulsar a un docente, $y$ dicha expectativa no se hace realidad, el grado de motivación para participar en estas encuestas bajará considerablemente. Es fundamental que los dos actores del acto pedagógico conozcan las reglamentaciones de la universidad sobre la evaluación docente, con el fin de comprender su propósito, alcances y limitaciones. Por ello es indispensable socializar estos documentos a toda la comunidad educativa y resaltar algunos de sus principios al inicio del formato de evaluación.

Hay que realizar un proceso pedagógico para explicar a los estudiantes el valor de la evaluación; en ese sentido, que tomen conciencia de que se hace la evaluación para mejorar y que los comentarios deben guiar al docente hacia lo que debería hacer, sugerir caminos más que descalificar o sancionar. Han de saber también que la docencia solo es una de las funciones del profesor universitario.

También con los docentes es necesario llevar a cabo un proceso pedagógico en el que se comprenda la importancia de la evaluación para ellos mismos, para los estudiantes, para el programa académico y para la universidad. La desconfianza del profesor frente a las evaluaciones debido a la poca objetividad que le atribuye, ha de combatirse, ya que baja sensiblemente el grado de receptividad de estas. Los profesores deben concebir la evaluación docente como una oportunidad de mejorar sus prácticas y sintonizar con sus estudiantes. Es necesario que vean la evaluación como un aprendizaje.

Así mismo, para evaluar adecuadamente es importante tener en cuenta las condiciones en las que se dicta y recibe una clase. La población, los recursos y el ambiente laboral son determinantes tanto en los procesos de enseñanza como de aprendizaje. El exceso de trabajo de profesores y estudiantes, que se traduce en la falta de tiempo, 
repercute negativamente en los procesos de enseñanza, aprendizaje y en la evaluación.

De igual manera, es preciso conciliar lo que se entiende por un buen profesor en el ámbito universitario. Ya Tejedor (2009) había indicado: "Puede resultarnos extraño tener que aceptar que, pese a los muchos estudios realizados no existe en la actualidad un consenso amplio sobre lo que es un 'buen profesor"' (p. 157).

Álvarez (s.f., p. 17), citando los trabajos de González Soler (1980), asevera que "los profesores más eficaces son aquellos que se muestran más humanos en el amplio sentido del término, es decir, agradables, afectuosos, justos, democráticos y más capaces para relacionarse con sus alumnos". Esto es lo que se encontró en esta investigación, como se mostró anteriormente: para los estudiantes un buen profesor es el que saber enseñar, el que logra que los alumnos lo entiendan. Las malas relaciones con el profesor son fuertemente castigadas en la evaluación docente. Este hallazgo coincide con lo planteado por Zambrano, Meda, y Lara (2005, p. 68) quienes señalan que factores subjetivos como las relaciones personales entre profesor y alumnos y "valoraciones externas de otros alumnos y profesores" determinan en gran medida las evaluaciones docentes.

De igual manera, es preciso reflexionar en torno al saber mismo que se transmite, consideración que está presente en un número importante de docentes ("el formato mide básicamente el cómo, no el qué") y en cambio aparece de manera marginal en los estudiantes. Si un buen profesor es el que explica bien, surge las preguntas: ¿Qué es explicar bien? ¿El que explica en forma sencilla? ¿Qué pasa con contenidos complejos? ¿Qué pasa con el lenguaje de una disciplina? Estudios como los de Carlino (2003), Ochoa y Cueva (2012) y Bazerman (1988) han mostrado que es función de la universidad adentrar a los estudiantes en los discursos propios de las disciplinas, lo que significa hablar en sus mismos términos. También es pertinente el texto de Zuleta (2005), El elogio de la dificultad. Si un buen maestro es el que simplifica todo, ¿qué pasa con los docentes que no son así?
De otra parte, ¿qué pasa con los procesos investigativos que instauran algunos profesores en sus aulas? ¿Debe la docencia tener una estrecha relación con la investigación? ¿Cómo? ¿Llevando los resultados de la investigación al aula o fomentando que los estudiantes realicen pequeñas microinvestigaciones? $¿$ No es buen docente el que "incentiva al estudiante a la investigación y al trabajo autónomo" y el que logra "crear una ruptura conceptual"? En los formatos no suele evaluarse actividades que realizan profesores, como incentivar la participación de los estudiantes en proyectos de investigación, en seminarios o congresos, o en publicaciones académicas.

Los estudiantes responsabilizan a los docentes por su baja motivación, pero desconocen la situación del profesor. ¿Qué pasa con la motivación de ellos? ¿Qué pasa con alumnos desidiosos, con alumnos que reclaman, pero no responden académicamente? ¿Qué pasa con instituciones donde el salario docente es un verdadero irrespeto a esta profesión? Estas y otras condiciones son desconocidas por estudiantes evaluadores.

El tema de la justicia aparece en ambas poblaciones entrevistadas. Los docentes piden una evaluación justa y los estudiantes señalan que, en ocasiones, son evaluados injustamente. Este sentimiento obstaculiza la evaluación docente, pues los profesores no aceptan las críticas y los estudiantes castigan a los profesores con quienes han obtenido malas notas. Nos preguntamos ¿cómo se consigue la objetividad y la justicia? Creemos que a través de una estrategia múltiple que sensibilice a los protagonistas sobre la importancia, alcance y límites de la evaluación, que revise continuamente qué se evalúa, que haya una correlación entre evaluación del docente y desempeños de los alumnos y que haya una autoevaluación tanto de parte del profesor como del estudiante que evalúa.

También se logra mayor objetividad cuando los formatos que se usan para evaluar y las estrategias que se implementan logran su propósito. Si bien los formatos virtuales tienen muchas virtudes, el hecho de que sea algo opcional restringe la participación del estudiantado. Sugerimos que, si la evaluación 
es virtual, se asigne un día para llenarla, un espacio y un tiempo determinados. De no ser esto posible, es preferible hacerla en las aulas de manera física, en papel, máximo cuando algunos entrevistados señalaron que no evaluaban por pereza, falta de tiempo u olvido. No es aconsejable que el proceso coincida con los exámenes finales.

Los formatos deben focalizar la atención de los estudiantes sobre los aspectos que se quieren evaluar. En esa medida, conviene que tengan una pequeña introducción donde se señale la importancia de la evaluación, el perfil de profesor de la universidad, los objetivos que se persiguen al evaluar y los alcances de la evaluación, y luego subtítulos que orienten sobre los macrotópicos por los que se indaga.

El hecho de que el formato sea anónimo permite una mayor libertad al estudiante para expresarse, pero este debe tomar conciencia de la responsabilidad de dicha libertad; especialmente, para el caso de instituciones educativas donde la evaluación de los estudiantes sí es un criterio para permanecer en un cargo, lo que desconoce claramente los planteamientos actuales de la evaluación que sugieren que se tengan en cuenta perspectivas diversas y que se ponga en el centro de la reflexión la motivación como elemento decisivo para los procesos de enseñanza, aprendizaje y evaluación. Al respecto, es muy grave que los alumnos reconozcan que, en ocasiones, se guían por rumores para evaluar a un profesor o que se ponen de acuerdo para evaluarlo negativamente.

La relación entre notas y evaluación docente amerita una reflexión por parte de la comunidad académica: ¿Qué tanta responsabilidad tiene el docente frente a las notas de los estudiantes? ¿Es la única variable que interviene? ¿Qué es ser un estudiante universitario?, ¿el que estudia?, ¿el que aprende? ¿Cuáles son las funciones de un profesor en este nivel? Llama la atención que los estudiantes responsabilicen a los profesores por sus bajos desempeños, pero no sienten que sus actuaciones académicas tienen relación con la calidad de la educación y la calidad de las prácticas docentes; por ejemplo, el rigor, compromiso y disciplina aca- démica, el diálogo que se puede entablar a tiempo con los docentes para encauzar procesos, etc.

Es muy útil la sugerencia de hacer un alto en el camino a mitad del semestre y dialogar con los estudiantes acerca de la marcha del curso. De esta manera se logra que la evaluación cumpla su cometido.

La baja motivación que manifiestan ambas poblaciones hacia la evaluación (poca participación, poca receptividad, falta de objetividad) y la motivación extrínseca que mueve a ambas poblaciones y su percepción de que la evaluación no tiene impacto es un hallazgo preocupante, con miras a lograr el objetivo de la evaluación docente. Ambas poblaciones no ven en la evaluación cumplidas sus expectativas, las cuales, además, son distintas, como ya se señaló.

A pesar de que algunos aspectos del formato se cuestionan, como el hecho de que no haya una interrelación entre el desempeño del estudiante y la evaluación que otorga, o el hecho de que el formato centra su atención en aspectos metodológicos y en aspectos personales, en detrimento del conocimiento propiamente dicho y en contravía de los objetivos de la educación superior, lo que amerita una revisión del formato; el problema más grave reside en no tener claridad sobre la función de dicha evaluación y en lo que sucede después de que se evalúa: no hay retroalimentación para los actores involucrados, no hay diálogo entre ellos que permita una reflexión sobre la función docente en la universidad, en el programa académico y en las asignaturas. Es esencial recordar, como lo señala Stobart (2010, p. 203), que las interpretaciones que se hagan de esos resultados son más cruciales que los mismos resultados; interpretaciones en las que deben participar estudiantes y profesores, y donde se debe reconocer como punto de partida las limitaciones de toda evaluación, la subjetividad que la caracteriza y el cuidado de emprender acciones represivas como consecuencia de ello.

En esta investigación se quiso recuperar las experiencias de esta población y darles voz con el presupuesto de que como actores principales de este proceso su mirada es vital y puede servir para reflexionar sobre las limitaciones del proceso y sus alcances. 
Las representaciones docentes expresan cómo se relacionan con la evaluación, lo que aprueban de ella (la evaluación misma, a los estudiantes como fuentes de información válida), lo que desaprueban (el hecho de que sea la única fuente de información, que se use con fines distintos a los formativos, el peso igualitario que se le da a las opiniones de todos los estudiantes, la relación entre calificaciones y evaluación y el privilegio de ciertos rasgos del docente, v. gr. metodología, puntualidad, relaciones personales, en detrimento de otros: investigación, conocimiento, motivación a la productividad) y su actitud (de reserva y desconfianza) .

En las representaciones de los estudiantes, la evaluación vincula rasgos de los profesores con la eficacia docente. Para esta población un buen profesor es quien maneja una adecuada metodología, motiva a los estudiantes, propicia un buen clima de aula y, en consecuencia, alcanza los objetivos propuestos.

Unas y otras representaciones orientan la forma como actúan profesores y estudiantes frente a la evaluación. Por ello, si se desea realizar algún cambio tanto al modelo de evaluación como a los instrumentos y aplicación de los mismos y si se desea investigar sobre algunos tópicos sugeridos en ellas (como la "buena enseñanza"), hay que atender a estas representaciones.

\section{Referencias}

Álvarez, F. (s.f.). ¿Qué hay que evaluar de los docentes? Recuperado de http://eoepsabi.educa.aragon.es/ descargas/H_Recursos/h_1_Psicol_Educacion/ h_1.4.Eval_desemp_docente/1.4.Que_evaluar_de_ los_docentes.pdf.

Andión, M. (2007). Sobre la calidad en la educación superior: una visión cualitativa. Reencuentro, 50, 83-92.

Arbesú, M. (2004). Evaluación de la docencia universitaria: Una propuesta alternativa que considera la participación de los profesores. RMIE, 9(23), 863-890.

Bazerman, Ch. (1988). Shaping Writing Knowledge, The Genre and Activiyt of the Experimental Article in Science. Madison, Wisconsin: University of Wisconsin Press.
Carlino, P. (2003). Alfabetización académica: un cambio necesario, algunas alternativas posibles. Educere, 6(20), 409-420.

Casero, A. (2008). Propuesta de un cuestionario de evaluación de la calidad docente universitaria consensuado entre alumnos y profesores. Revista de Investigación Educativa, 26(1), 25-44.

Congreso de la República de Colombia. Ley 30 de diciembre 28 de 1992. Recuperado de www.cna.gov.co/1741/ articles-186370_ley_3092.pdf

Echeverry, J. (2011). Evaluación docente integral con fines de mejoramiento. EDIFICANDO. Bogotá: Universidad Nacional de Colombia. Recuperado de http:// www.edificando.unal.edu.co/descargables/modeledificando.pdf.

Fernández, J., Mateo. M. y Muñiz, J. (1996). Valoración por parte del profesorado de la evaluación docente realizada por los alumnos. Psicothema, 8(1), 167-172.

Fuentes, M. y Herrero, J. (1999). Evaluación docente: hacia una fundamentación de la autoevaluación. Revista Electrónica Interuniversitaria de Formación del Profesorado, 2(1), 353-367.

Moscovici, S. (1985). Psicología social I. Barcelona: Paidós.

Ochoa, L. y Cueva. L. (2012). Tesis y deserción: entre el compromiso y el obstáculo. Bogotá: Universidad Nacional de Colombia.

Stobart, S. (2010). Tiempos de pruebas los usos y abusos de la evaluación. Madrid: Ediciones Morata.

Tejedor, F. (2009). Evaluación del profesorado universitario: enfoque metodológico y algunas aportaciones de la investigación. Estudios sobre Educación, 16, 79-102.

Universidad del Rosario (2012). Lineamientos institucionales para la evaluación integral de los profesores de la Universidad del Rosario. Bogotá. Recuperado de http://www.urosario.edu.co/Profesores/ur/ Evaluacion-Integral/Politicas-y-Lineamientos/ lineamientos/

Vásquez, F. y Gabalán, J. (2012). La evaluación docente en posgrado: variables y factores influyentes. Educ., 15(3), 445-460.

Villa, A. (1985). La evaluación del profesor: perspectivas y resultados. Revista de Educación, 277, 55-93.

Villa, A. (2008). La excelencia docente. Revista de Educación, núm. extraordinario, 172- 212. 
Universidad Pedagógica Nacional

Facultad de Humanidades

Zambrano, R., Meda, R.M. y Lara, B. (2005). Evaluación de profesores universitarios por parte de los alumnos mediante el Cuestionario de evaluación de Desempeño Docente (CEDED). Revista de Educación $y$ Desarrollo, 4, 63-69.

Zuleta, E. (2005). El elogio de la dificultad y otros ensayos. Medellín: Hombre Nuevo Editores y Fundación Estanislao Zuleta. 